\title{
ON THE EXISTENCE OF HURWITZ POLYNOMIALS WITH NO HADAMARD FACTORIZATION*
}

\author{
STANISŁAW BIAŁAS ${ }^{\dagger}$ AND MICHA£ GÓRA ${ }^{\ddagger}$
}

\begin{abstract}
A Hurwitz stable polynomial of degree $n \geq 1$ has a Hadamard factorization if it is a Hadamard product (i.e., element-wise multiplication) of two Hurwitz stable polynomials of degree $n$. It is known that Hurwitz stable polynomials of degrees less than four have a Hadamard factorization. It is shown that, for arbitrary $n \geq 4$, there exists a Hurwitz stable polynomial of degree $n$ which does not have a Hadamard factorization.
\end{abstract}

Key words. Hadamard factorization, Hadamard product of polynomials, Hurwitz stable polynomials.

AMS subject classifications. 26C10, 30C15, 93D99.

1. Introduction. A polynomial is said to be (Hurwitz) stable if all its zeros lie in the open left-half of the complex plane. In the entire class of polynomials, stable polynomials occupy a special place and stability of polynomials is a classical topic having both theoretical and applied significance.

In this short note, we deal with the Hadamard factorization problem. Recall, that a Hurwitz stable polynomial of degree $n \geq 1$ has (or admits) a Hadamard factorization if it is a Hadamard product (i.e., element-wise multiplication) of two Hurwitz stable polynomials of degree $n$. A problem of the existence of a Hadamard factorization for a given stable polynomial has been taken by many authors. It is obvious that $\mathcal{F}_{1}^{+}=\mathcal{H}_{1}^{+}$and $\mathcal{F}_{2}^{+}=\mathcal{H}_{2}^{+}$, where $\mathcal{H}_{n}^{+}$denotes the entire family of Hurwitz stable polynomials of degree $n$ with positive coefficients and $\mathcal{F}_{n}^{+}$is its subset containing only polynomials admitting a Hadamard factorization. Moreover, as follows from Garloff and Shrinivasan [3], we know that $\mathcal{F}_{3}^{+}=\mathcal{H}_{3}^{+}$but $\mathcal{F}_{4}^{+} \neq \mathcal{H}_{4}^{+}$. Some necessary and necessary and sufficient conditions for the existence of a Hadamard factorization for a polynomial of arbitrary degree can be found in Loredo-Villalobos and Aguirre-Hernández [6, 7] (unfortunately, their conditions cannot be effectively applied in practice) and some topological properties of the set $\mathcal{F}_{n}^{+}$(as openness, non-convexity and arc-connectedness) in Aguirre-Hernández et al. [1].

Note also, that there are some issues in which polynomials admitting a Hadamard factorization play an important role. For example, in [2], the authors of this paper have considered the generalized Hadamard product of polynomials and have shown, among others, that if $f$ is stable and $g$ has a Hadamard factorization then the generalized Hadamard product of $f$ and $g$ is stable (in [2], one can also find a very simple sufficient condition for the Hadamard factorizability of a polynomial). Taking into account all these results it seems to be quite surprising that we still do not know if there exists a polynomial of an arbitrary degree greater than four which does not admit a Hadamard factorization. We will show that this is the case.

\footnotetext{
*Received by the editors on November 19, 2019. Accepted for publication on March 7, 2020. Handling Editor: Froilán M. Dopico. Corresponding Author: Michał Góra.

${ }_{\dagger}$ The School of Banking and Management, ul. Armii Krajowej 4, 30-150 Kraków, Poland (sbialas@agh.edu.pl).

${ }^{\ddagger}$ AGH University of Science and Technology, Faculty of Applied Mathematics, al. Mickiewicza 30, 30-059 Kraków, Poland (gora@agh.edu.pl). The research work of this author was partially supported by the Faculty of Applied Mathematics AGH UST statutory tasks (grant no. 11.11.420.004) within subsidy of Ministry of Science and Higher Education.
} 
2. Preliminary results. In this part, we introduce the basic notation and remind some results which will be used in the sequel.

2.1. Basic notations. We use standard notation: $\mathbb{N}$ and $\mathbb{R}$ stand for the set of positive integers and real numbers, respectively; $\mathbb{R}^{n \times n}$ stands for the set of real matrices of order $n \times n$; $\pi_{n}^{+}$denotes the family of $n$-th degree polynomials with positive coefficients.

2.2. Stable polynomials. A polynomial $f \in \pi_{n}^{+}(n \geq 1)$,

$$
f(s)=a_{0}+a_{1} s+\cdots+a_{n-1} s^{n-1}+a_{n} s^{n},
$$

is Hurwitz stable (or shortly stable) if all its zeros have negative real parts. It is well known (and easily verified) that a necessary condition for the stability of a real polynomial is that its coefficients are all of the same sign; without losing generality we will assume in the sequel that they are positive.

Let $H_{f} \in \mathbb{R}^{n \times n}$ be the Hurwitz matrix associated with polynomial (2.1), i.e.,

$$
H_{f}=\left(\begin{array}{cccccc}
a_{n-1} & a_{n} & 0 & 0 & \cdots & 0 \\
a_{n-3} & a_{n-2} & a_{n-1} & a_{n} & \cdots & 0 \\
a_{n-5} & a_{n-4} & a_{n-3} & a_{n-2} & \cdots & 0 \\
\vdots & \vdots & a_{n-5} & a_{n-4} & \cdots & 0 \\
\vdots & \vdots & \vdots & \vdots & \ddots & \vdots \\
0 & 0 & 0 & 0 & \cdots & a_{0}
\end{array}\right)
$$

From among many interesting properties of the Hurwitz matrix we recall the following one given by Kemperman [5] (see Theorem 2 therein).

THEOREM 2.1. If $f \in \mathcal{H}_{n}^{+}$then every square submatrix of the Hurwitz matrix $H_{f}$ has positive determinant if and only if all its diagonal elements are positive.

Note that as long as $n \geq 4$, the matrix

$$
\left(\begin{array}{ccc}
a_{3} & a_{4} & 0 \\
a_{1} & a_{2} & a_{n-1} \\
0 & a_{0} & a_{n-3}
\end{array}\right)
$$

is a submatrix of matrix (2.2). Thus, in view of Theorem 2.1, it allows us to conclude that if polynomial (2.1) of degree $n \geq 4$ is stable then

$$
a_{3} a_{2}-a_{1} a_{4}>0, \quad a_{n-3} a_{2}-a_{n-1} a_{0}>0
$$

and

$$
a_{n-3} a_{3} a_{2}-a_{n-3} a_{4} a_{1}-a_{n-1} a_{3} a_{0}>0 .
$$

These inequalities will be crucial in our further considerations. 
2.3. Polynomials admitting a Hadamard factorization. Together with polynomial $f$ of the form (2.1) we will consider a polynomial $g \in \pi_{n}^{+}$,

$$
g(s)=b_{0}+b_{1} s+\cdots+b_{n-1} s^{n-1}+b_{n} s^{n}
$$

their Hadamard product $f \circ g \in \pi_{n}^{+}$defined as an element-wise multiplication, i.e.,

$$
(f \circ g)(s)=a_{0} b_{0}+a_{1} b_{1} s+\cdots+a_{n-1} b_{n-1} s^{n-1}+a_{n} b_{n} s^{n}
$$

and their Hadamard quotient $f \diamond g \in \pi_{n}^{+}$defined as an element-wise division, i.e.,

$$
(f \diamond g)(s)=\frac{a_{0}}{b_{0}}+\frac{a_{1}}{b_{1}} s+\cdots+\frac{a_{n-1}}{b_{n-1}} s^{n-1}+\frac{a_{n}}{b_{n}} s^{n} .
$$

Following Garloff and Wagner [4], we say that the polynomial $f \in \mathcal{H}_{n}^{+}$has a Hadamard factorization if there exist two polynomials $f_{1}, f_{2} \in \mathcal{H}_{n}^{+}$such that $f=f_{1} \circ f_{2}$. Equivalently, the polynomial $f \in \mathcal{H}_{n}^{+}$has a Hadamard factorization if there exists a polynomial $g \in \mathcal{H}_{n}^{+}$such that $f \diamond g \in \mathcal{H}_{n}^{+}$.

3. Main result. Let $f \in \pi_{n}^{+}$be as in (2.1), and let $\delta_{1}(f)$ and $\delta_{2}(f)$ be two positive numbers given by

$$
\delta_{1}(f)=\frac{a_{1} a_{4}}{a_{3} a_{2}} \quad \text { and } \quad \delta_{2}(f)=\frac{a_{n-1} a_{0}}{a_{n-3} a_{2}}
$$

We start with two simple observations.

LEMma 3.1. For every $n \geq 4$ and for the polynomial $f \in \mathcal{H}_{n}^{+}$we have

$$
\delta_{1}(f)<1, \quad \delta_{2}(f)<1
$$

and

$$
\delta_{1}(f)+\delta_{2}(f)<1
$$

Proof. The above inequalities follow from the stability of $f$ : conditions (3.8) and (3.9) are equivalent to (2.4) and (2.5), respectively.

LEMMA 3.2. If for $f, g \in \pi_{n}^{+}$we have $f \diamond g \in \mathcal{H}_{n}^{+}$, then

$$
\delta_{1}(f)<\delta_{1}(g) \quad \text { and } \quad \delta_{2}(f)<\delta_{2}(g)
$$

Proof. It is easy to see that

$$
\delta_{1}(f \diamond g)=\frac{\delta_{1}(f)}{\delta_{1}(g)} \quad \text { and } \quad \delta_{2}(f \diamond g)=\frac{\delta_{2}(f)}{\delta_{2}(g)}
$$

Then, the thesis is a simple consequence of Lemma 3.1.

We are now ready to prove the main result of this work.

Theorem 3.3. For every $n \geq 4$ there exists a stable polynomial of degree $n$ that does not have a Hadamard factorization. 
Proof. Fix any $n \geq 4$ and suppose, by contradiction, that every stable polynomial of degree $n$ admits a Hadamard factorization. It implies that for an arbitrary $g_{0} \in \mathcal{H}_{n}^{+}$, there exists $g_{1} \in \mathcal{H}_{n}^{+}$such that $g_{0} \diamond g_{1} \in \mathcal{H}_{n}^{+}$. Repeating this reasoning one can show that for $g_{i} \in \mathcal{H}_{n}^{+}$there exists $g_{i+1} \in \mathcal{H}_{n}^{+}$such that $g_{i} \diamond g_{i+1} \in \mathcal{H}_{n}^{+}$. In this way we obtain a sequence of stable polynomials $\left\{g_{i}\right\}$ which generates two sequences of positive numbers $\left\{\delta_{1}\left(g_{i}\right)\right\}$ and $\left\{\delta_{2}\left(g_{i}\right)\right\}$ (as in (3.7)). It follows from Lemma 3.2 that sequences $\left\{\delta_{1}\left(g_{i}\right)\right\}$ and $\left\{\delta_{2}\left(g_{i}\right)\right\}$ are increasing. Moreover, by Lemma 3.1, they are bounded from above and thus convergent. They are also bounded from below by zero, and thus, their limits are positive. It means that

$$
\frac{\delta_{1}\left(g_{i}\right)}{\delta_{1}\left(g_{i+1}\right)}+\frac{\delta_{2}\left(g_{i}\right)}{\delta_{2}\left(g_{i+1}\right)} \rightarrow 2
$$

as $i \rightarrow \infty$. On the other hand, according to Lemma 3.1 it follows from the stability of $g_{i} \diamond g_{i+1}$ that

$$
\frac{\delta_{1}\left(g_{i}\right)}{\delta_{1}\left(g_{i+1}\right)}+\frac{\delta_{2}\left(g_{i}\right)}{\delta_{2}\left(g_{i+1}\right)}=\delta_{1}\left(g_{i} \diamond g_{i+1}\right)+\delta_{2}\left(g_{i} \diamond g_{i+1}\right)<1
$$

for every $i \in \mathbb{N}$. This contradicts to (3.10) completing the proof.

Garloff and Shrinivasan proved in [3] that there exists a polynomial of degree 4 which is not a Hadamard product of stable polynomials of degree 4 . Theorem 3.3 shows that similar examples can be constructed for every $n \geq 4$.

4. Concluding remarks and open problems. We have considered the Hadamard factorization problem for real polynomials. We have proved that for an arbitrary $n \geq 4$ there exists a Hurwitz stable polynomial of degree $n$ which does not have a Hadamard factorization. Unfortunately, the proof of our main result is not constructive, that is, it does not tell us how to construct such a polynomial. An effective method of finding polynomials that do not admit a Hadamard factorization seems to be a challenging open problem.

Other open problems in this area are the development of necessary and sufficient conditions for the Hadamard factorizability of a polynomial as well as to develop factorization (in the Hadamard sense) algorithms for stable polynomials admitting a Hadamard factorization.

Acknowledgments. The authors wish to thank Prof. Froilán Dopico for his help during the review process.

\section{REFERENCES}

[1] B. Aguirre-Hernández, E.C. Díaz-González, C.A. Loredo-Villalobos, and F.R. García-Sosa. Properties of the set of Hadamardized Hurwitz polynomials. Math. Probl. Eng., 2015:Article 695279, doi: 10.1155/2015/695279, 2015.

[2] S. Białas and M. Góra. The generalized Hadamard product of polynomials and its stability. Linear Multilinear Algebra, to appear, doi: 10.1080/03081087.2019.1627277.

[3] J. Garloff and B. Shrinivasan. The Hadamard factorization of Hurwitz and Schur stable polynomials. In: R. Jeltsch and M. Mansour (editors), Stability Theory, Birkhäusee, 19-21, 1996.

[4] J. Garloff and D.G. Wagner. Hadamard product of stable polynomials are stable. J. Math. Anal. Appl., 202:797-808, 1996.

[5] J.H.B. Kemperman. A Hurwitz matrix is totally positive. SIAM J. Math. Anal., 13(2):331-341, 1982.

[6] C.A. Loredo-Villalobos and B. Aguirre-Hernández. Hadamard factorization of stable Polynomials. Dyn. Contin. Discrete Impuls. Syst. Ser. A Math. Anal., 22:325-332, 2015.

[7] C.A. Loredo-Villalobos and B. Aguirre-Hernández. Necessary conditions for Hadamard factorizations of Hurwitz polynomials. Automatica, 47:1409-1413, 2011. 